\title{
DOUBLE SEQUENCE CORE THEOREMS
}

\author{
RICHARD F. PATTERSON
}

(Received 13 April 1998 and in revised form 1 September 1998)

\begin{abstract}
In 1900, Pringsheim gave a definition of the convergence of double sequences. In this paper, that notion is extended by presenting definitions for the limit inferior and limit superior of double sequences. Also the core of a double sequence is defined. By using these definitions and the notion of regularity for 4-dimensional matrices, extensions, and variations of the Knopp Core theorem are proved.
\end{abstract}

Keywords and phrases. Core of a sequence, double sequence, regular matrix, P-convergent.

1991 Mathematics Subject Classification. Primary 40B05; Secondary $40 \mathrm{C} 05$.

1. Introduction. The notion of convergence for double sequences was presented by Pringsheim. Also, in $[2,3,4,5,10]$ the 4-dimensional matrix transformation $(A x)_{m, n}=$ $\sum_{k, l=0,0}^{\infty, \infty} a_{m, n, k, l} x_{k, l}$ was studied extensively by Robison and Hamilton. In their work and throughout this paper, the 4-dimensional matrices and double sequences have complex-valued entries unless specified otherwise. In this paper, we extend the notion of convergence by defining new double sequence spaces and consider the behavior of 4-dimensional matrix transformations on our new spaces. We also present definitions for limit inferior/limit superior of a double sequence, regularity of a 4-dimensional matrix, and the core of a double sequence. Using these definitions and the notion of regularity for a 4-dimensional matrix, we present multidimensional analogues to the Knopp Core theorem. We also present extensions and variations of this theorem.

\section{Definitions and preliminary results}

Definition 2.1 [Pringsheim, 1900]. A double sequence $[x]$ has Pringsheim limit $L$ (denoted by $\mathrm{P}-\lim [x]=L$ ) provided that given $\epsilon>0$ there exists $N \in \mathbf{N}$ such that $\mid x_{k, l}-$ $L \mid<\epsilon$ whenever $k, l>N$. We shall describe such an $[x]$ more briefly as "P-convergent."

A double sequence $[x]$ is bounded if and only if there exists a positive number $M$ such that $\left|x_{k, l}\right|<M$ for all $k$ and $l$ (which shall be denoted by $[|x|]<M$ ). Note that a convergent double sequence need not be bounded. In 1900, Pringsheim gave the following definition: a double sequence $[x]$ is called definite divergent if for every (arbitrarily large) $G>0$ there exist two natural numbers $n_{1}$ and $n_{2}$ such that $\left|x_{n, k}\right|>G$ for $n \geq n_{1}, k \geq n_{2}$. This definition is clearly equivalent to P- $\lim [|x|]=\infty$.

DefinITION 2.2. The sequence $[y]$ is a subsequence of the double sequence $[x]$ provided that there exist two increasing double index sequences $\left\{n_{j}^{i}\right\}$ and $\left\{k_{j}^{i}\right\}$ such that $n_{0}^{1}=k_{0}^{1}=n_{-1}^{0}=k_{-1}^{0}=0$ and 
$n_{1}^{i} \& k_{1}^{i}$ are both chosen such that $\max \left\{n_{2 i-3}^{i-1}, k_{2 i-3}^{i-1}\right\}<n_{1}^{i} \& k_{1}^{i}$, $n_{2}^{i} \& k_{2}^{i}$ are both chosen such that $\max \left\{n_{1}^{i}, k_{1}^{i}\right\}<n_{2}^{i} \& k_{2}^{i}$,

$n_{3}^{i} \& k_{3}^{i}$ are both chosen such that $\max \left\{n_{2}^{i}, k_{2}^{i}\right\}<n_{3}^{i} \& k_{3}^{i}$,

$n_{2 i-1}^{i} \& k_{2 i-1}^{i}$ are both chosen such that $\max \left\{n_{2(i-1)}^{i}, k_{2(i-1)}^{i}\right\}<n_{2 i-1}^{i} \& k_{2 i-1}^{i}$, with

$$
\begin{gathered}
y_{1, i}=x_{n_{1}^{i}, k_{1}^{i}}, \\
y_{2, i}=x_{n_{2}^{i}, k_{2}^{i},} \\
y_{3, i}=x_{n_{3}^{i}, k_{3}^{i}}, \\
\vdots \\
y_{i, i}=x_{n_{i}^{i}, k_{i}^{i}}, \\
y_{i, i+1}=x_{n_{i+1}^{i}, k_{i+1}^{i}}, \\
\vdots \\
y_{i, 2 i-1}=x_{n_{2 i-1}^{i}, k_{2 i-1}^{i}}
\end{gathered}
$$

for $i=1,2,3, \ldots$.

EXAMPLE 2.1. The double sequences whose $n$, $k$-terms are $y_{n, k}=1$ and $z_{n, k}=-1$ for each $n$ and $k$ are both subsequences of the double sequence whose $n, k$ th term is $x_{n, k}=(-1)^{n+k}$. Indeed, every double sequence of 1's and -1 's is a subsequence of this $[x]$.

A two dimensional matrix transformation is said to be regular if it maps every convergent sequence into a convergent sequence with the same limit. In 1926, Robison presented a 4-dimensional analogue of regularity for double sequences in which he added an additional assumption of boundedness: a 4-dimensional matrix $A$ is said to be RHregular if it maps every bounded P-convergent sequence into a P-convergent sequence with the same P-limit.

The following is a 4-dimensional analogue of the well-known Silverman-Toeplitz theorem [6].

THEOREM 2.1 (Hamilton [2], Robison [10]). The 4-dimensional matrix A is RH-regular if and only if

$\left(\mathrm{RH}_{1}\right) \mathrm{P}-\lim _{m, n} a_{m, n, k, l}=0$ for each $k$ and $l$;

$\left(\mathrm{RH}_{2}\right) \mathrm{P}-\lim _{m, n} \sum_{k, l=0,0}^{\infty, \infty} a_{m, n, k, l}=1$;

$\left(\mathrm{RH}_{3}\right) \mathrm{P}-\lim _{m, n} \sum_{k=0}^{\infty}\left|a_{m, n, k, l}\right|=0$ for each $l$;

$\left(\mathrm{RH}_{4}\right) \mathrm{P}-\lim _{m, n} \sum_{l=0}^{\infty}\left|a_{m, n, k, l}\right|=0$ for each $k$;

$\left(\mathrm{RH}_{5}\right) \sum_{k, l=0,0}^{\infty, \infty}\left|a_{m, n, k, l}\right|$ is $\mathrm{P}$-convergent; and

$\left(\mathrm{RH}_{6}\right)$ there exist positive numbers $A$ and $B$ such that $\sum_{k, l>B}\left|a_{m, n, k, l}\right|<A$.

Definition 2.3. A number $\beta$ is called a Pringsheim limit point of the double sequence $[x]$ provided that there exists a subsequence $[y]$ of $[x]$ that has Pringsheim $\operatorname{limit} \beta: \mathrm{P}-\lim [y]=\beta$. 
REMARK 2.1. The definition of a Pringsheim limit point is equivalent to the following statement: $\beta$ is a Pringsheim limit point of $[x]$ if and only if there exist two increasing index sequences $\left\{n_{i}\right\}$ and $\left\{k_{i}\right\}$ such that $\lim _{i} x_{n_{i}, k_{i}}=\beta$. A double sequence $[x]$ is divergent in the Pringsheim sense (P-divergent) provided that $[x]$ is not $\mathrm{P}$-convergent. This is equivalent to the following: a double sequence $[x]$ is P-divergent if and only if either $[x]$ contains two subsequences with distinct finite limit points or $[x]$ contains an unbounded subsequence. Also note that, if $[x]$ contains an unbounded subsequence then $[x]$ also contains a definite divergent subsequence.

In [7] Knopp introduced the concept of the core a complex number sequence. We follow that idea in defining the core of a double sequence.

DEFINITION 2.4. Let P- $C_{n}\{x\}$ be the least closed convex set that includes all points $x_{k, l}$ for $k, l>n$; then the Pringsheim core of the double sequence $[x]$ is the set $\mathrm{P}-C\{x\}=\bigcap_{n=1}^{\infty}\left[\mathrm{P}-C_{n}\{x\}\right]$.

THEOREM 2.2 [Knopp, 1930]. If $A$ is a nonnegative regular matrix then the core of $[A x]$ is contained in core of $[x]$, provided that $[A x]$ exists.

3. Main results. In a manner similar to the classical definitions of the limit superior and the limit inferior of a sequence, we present definitions for the limit superior and the limit inferior of a double sequence. Using these definitions one can characterize the Pringsheim core of a real-valued double sequence as the closed interval [P-liminf $x, \mathrm{P}-\lim \sup x]$.

DEFINITION 3.1. Let $[x]=\left\{x_{k, l}\right\}$ be a double sequence of real numbers and for each $n$, let $\alpha_{n}=\sup _{n}\left\{x_{k, l}: k, l \geq n\right\}$. The Pringsheim limit superior of $[x]$ is defined as follows:

(1) if $\alpha=+\infty$ for each $n$, then P-limsup $[x]:=+\infty$;

(2) if $\alpha<\infty$ for some $n$, then P-limsup $[x]:=\inf _{n}\left\{\alpha_{n}\right\}$.

Similarly, let $\beta_{n}=\inf _{n}\left\{x_{k, l}: k, l \geq n\right\}$ then the Pringsheim limit inferior of $[x]$ is defined as follows:

(1) if $\beta_{n}=-\infty$ for each $n$, then P-liminf $[x]:=-\infty$;

(2) if $\beta_{n}>-\infty$ for some $n$, then P-liminf $[x]:=\sup _{n}\left\{\beta_{n}\right\}$.

EXAMPLE 3.1. The following is an example of an $[x]$ which is neither bounded above nor bounded below; however, the Pringsheim limit superior and inferior are both finite numbers

$$
x_{k, l}:= \begin{cases}k, & \text { if } l=0, \\ -l, & \text { if } k=0, \\ (-1)^{k}, & \text { if } l=k>0, \\ 0, & \text { otherwise; }\end{cases}
$$

thus P-liminf $[x]=-1$ and P-limsup $[x]=1$.

The proof of the following proposition is the same as the proof for single dimensional sequences and is therefore left to the reader.

Proposition 3.1. If $[x]$ is a real-valued double sequence then

(1) $\mathrm{P}-\liminf [x] \leq \mathrm{P}-\limsup [x]$; 
(2) $\mathrm{P}-\lim [x]=L$ if and only if $\mathrm{P}-\lim \sup [x]=\mathrm{P}-\liminf [x]=L$;

(3) $\mathrm{P}-\lim \sup [-x]=-(\mathrm{P}-\liminf [x])$;

(4) $\mathrm{P}-\lim \sup ([x]+[y]) \leq(\mathrm{P}-\lim \sup [x])+(\mathrm{P}-\lim \sup [y])$;

(5) $\mathrm{P}-\liminf ([x]+[y]) \geq(\mathrm{P}-\liminf [x])+(\mathrm{P}-\liminf [y])$;

(6) if $[y]$ is a subsequence of the double sequence $[x]$ then

$$
\mathrm{P}-\liminf [x] \leq \mathrm{P}-\liminf [y] \leq \mathrm{P}-\lim \sup [y] \leq \mathrm{P}-\lim \sup [x]
$$

THEOREM 3.1. If $A$ is a nonnegative RH-regular summability matrix, then $\mathrm{P}-C\{A x\}$ $\subseteq \mathrm{P}-C\{x\}$ for any bounded sequence $[x]$ for which $[A x]$ exists.

Proof. Note that if $\mathrm{P}-C\{x\}$ is the complex plane then the result is trivial. We shall establish our theorem by considering separately the cases where $[x]$ is bounded or unbounded. In both cases the result will be established by proving the following: if there exists a $q$ such that for $\omega \notin \mathrm{P}-C_{q}\{x\}$, then there exists a $p$ such that $\omega \notin$ $\mathrm{P}-C_{p}\{A x\}$. When $[x]$ is bounded, $\mathrm{P}-C\{x\}$ is not the complex plane, thus there exists an $\omega \notin \mathrm{P}-C\{x\}$. This implies that there exists a $q$ for which $\omega \notin \mathrm{P}-C_{n}\{x\}$. Since $\omega$ is finite, we may assume that $\omega=0$ by the linearity of $A$. Since we are also given that $\mathrm{P}-C_{q}\{x\}$ is a convex set, we can rotate $\mathrm{P}-C_{q}\{x\}$ so that the distance from zero to P- $C_{q}\{x\}$ is the minimum of $\left\{|y|: y \in \mathrm{P}-C_{q}\{x\}\right\}$ and is on the positive real axis; say that this minimum is $3 d$. Since P- $C_{q}\{x\}$ is convex, all points of $\mathrm{P}-C_{q}\{x\}$ have a real part which is at least $3 d$. Let $M=\max \left\{\left|x_{k, l}\right|\right\}$. By the regularity conditions $\left(\mathrm{RH}_{1}\right)-\left(\mathrm{RH}_{4}\right)$ and the assumption $a_{m, n, k, l} \geq 0$, there exists an $N$ such that for $m, n>N$ the following holds:

$$
\begin{array}{ll}
\sum_{k, l \in I_{1}} a_{m, n, k, l}<\frac{d}{3 M}, & \sum_{k, l \in I_{2}} a_{m, n, k, l}<\frac{d}{3 M}, \\
\sum_{k, l \in I_{3}} a_{m, n, k, l}<\frac{d}{3 M}, & \sum_{k, l \in I_{4}} a_{m, n, k, l}>\frac{2}{3},
\end{array}
$$

where

$$
\begin{aligned}
& I_{1}=\left\{(k, l): 0 \leq k \leq k_{0} \& 0 \leq l \leq l_{0}\right\}, \\
& I_{2}=\left\{(k, l): k_{0}<k<\infty \& 0 \leq l<l_{0}\right\}, \\
& I_{3}=\left\{(k, l): 0<k \leq k_{0} \& l_{0}<l<\infty\right\}, \\
& I_{4}=\left\{(k, l): k_{0}<k<\infty \& l_{0}<l<\infty\right\} .
\end{aligned}
$$

Therefore for $m, n>N$

$$
\begin{aligned}
\mathfrak{R}\left\{\sum_{k, l=0,0}^{\infty, \infty} a_{m, n, k, l} x_{k, l}\right\}= & \mathfrak{R}\left\{\sum_{k, l \in I_{1}} a_{m, n, k, l} x_{k, l}\right\}+\mathfrak{R}\left\{\sum_{k, l \in I_{2}} a_{m, n, k, l} x_{k, l}\right\} \\
& +\mathfrak{R}\left\{\sum_{k, l \in I_{3}} a_{m, n, k, l} x_{k, l}\right\}+\mathfrak{R}\left\{\sum_{k, l \in I_{4}} a_{m, n, k, l} x_{k, l}\right\} \\
> & -M \sum_{k, l \in I_{1}} a_{m, n, k, l}-M \sum_{k, l \in I_{2}} a_{m, n, k, l} \\
& -M \sum_{k, l \in I_{3}} a_{m, n, k, l}+3 d \sum_{k, l \in I_{4}} a_{m, n, k, l}>-M \frac{3 d}{3 M}+3 d \frac{2}{3}=d .
\end{aligned}
$$


Therefore, $\mathfrak{R}\{A x\}>d$ which implies that there exists a $p$ for which $\omega=0$ is also outside of $\mathrm{P}-C_{p}\{A x\}$. Now suppose that $[x]$ is unbounded; the $\omega$ may be the point at infinity or not. If $\omega$ is not the point at infinity then choose $N$ such that for $m, n>N$ the following holds:

$$
\sum_{k, l \in I_{1}} a_{m, n, k, l}<\frac{d}{3 M}, \quad \sum_{k, l \in I_{2} \cup I_{3} \cup I_{4}} a_{m, n, k, l}>\frac{2}{3} .
$$

In a manner similar to the first part we obtain $\mathfrak{R}\{A x\}>d$. In the case when $\omega$ is the point at infinity, P- $C_{q}\{x\}$ is bounded for all $q$, which implies that $x_{k, l}$ is bounded for $k, l>q$. We may assume that $[|x|]<B$ for some positive number $B$ without loss of generality. Thus for $m$ and $n$ large we obtain the following:

$$
\left|\sum_{k, l=0,0}^{\infty, \infty} a_{m, n, k, l} x_{k, l}\right| \leq \sum_{k, l=0,0}^{\infty, \infty} a_{m, n, k, l}\left|x_{k, l}\right| \leq B \sum_{k, l=0,0}^{\infty, \infty} a_{m, n, k, l}<\infty .
$$

Hence, there exists a $p$ such that the point at infinity is outside of $\mathrm{P}-C_{p}\{A x\}$. This completes the proof of our theorem.

The following lemma is a multidimensional analogue of a lemma of Agnew in [1]. We use this lemma to prove Theorem 3.2, below.

LEMMA 3.1. If $\left\{a_{m, n, k, l}\right\}_{k, l=0,0}^{\infty, \infty}$ is a real or complex-valued 4-dimensional matrix such that $\left(\mathrm{RH}_{1}\right),\left(\mathrm{RH}_{3}\right),\left(\mathrm{RH}_{4}\right)$, and $\mathrm{P}-\limsup \sup _{m, n} \sum_{k, l=0,0}^{\infty, \infty}\left|a_{m, n, k, l}\right|=M$ hold, then for any bounded double sequence $[x]$ we obtain the following:

$$
\mathrm{P}-\lim \sup [|y|] \leq M(\mathrm{P}-\lim \sup [|x|]),
$$

where

$$
y_{m, n}=\sum_{k, l=0,0}^{\infty, \infty} a_{m, n, k, l} x_{k, l} .
$$

In addition, there exists a real-valued double sequence $[x]$ such that if $a_{m, n, k, l}$ is real with $0<\mathrm{P}$-limsup $[|x|]<\infty$ then

$$
\mathrm{P}-\lim \sup [|y|]=M(\mathrm{P}-\lim \sup [|x|]) \text {. }
$$

Proof. Let $[x]$ be bounded and define

$$
B:=\mathrm{P}-\lim \sup [|x|]<\infty .
$$

Given $\epsilon>0$ we can choose an $N$ such that $\left|x_{k, l}\right|<(B+\epsilon) / 3$ for each $k$, and/or $l>N$. Thus,

$$
\begin{aligned}
\left|y_{m, n}\right| \leq & \sum_{k, l=0,0}^{N, N}\left|a_{m, n, k, l}\right|\left|x_{k, l}\right|+\sum_{\substack{0 \leq l \leq N, N<k<\infty}}\left|a_{m, n, k, l}\right|\left|x_{k, l}\right| \\
& +\sum_{\substack{N<l \leq \infty, 0 \leq k \leq N}}\left|a_{m, n, k, l}\right|\left|x_{k, l}\right|+\sum_{\substack{k, l=N+1, N+1 \\
\leq}}^{\infty, a_{m, n, k, l}|| x_{k, l} \mid} \\
\leq & \sum_{k, l=0,0}^{N, N}\left|a_{m, n, k, l}\right|\left|x_{k, l}\right|+\sum_{\substack{0 \leq l \leq N, N<k<\infty}}\left|a_{m, n, k, l}\right|\left(\frac{B+\epsilon}{3}\right) \\
& +\sum_{\substack{N<l \leq \infty, 0 \leq k \leq N}}\left|a_{m, n, k, l}\right|\left(\frac{B+\epsilon}{3}\right)+\sum_{k, l=N+1, N+1}^{\infty, \infty}\left|a_{m, n, k, l}\right|\left(\frac{B+\epsilon}{3}\right),
\end{aligned}
$$


which yields

$$
\mathrm{P}-\lim \sup [|y|] \leq M(B+\epsilon) .
$$

Therefore the following holds:

$$
\mathrm{P}-\lim \sup [|y|] \leq M(\mathrm{P}-\lim \sup [|x|]) .
$$

Since

$$
\text { P- } \limsup _{m, n} \sum_{k, l=0,0}^{\infty, \infty}\left|a_{m, n, k, l}\right|=M,
$$

we may assume that $M>0$ without loss of generality. Using the RH-regularity conditions we choose $m_{0}, n_{0}, l_{0}$, and $k_{0}$, so large that

$$
\begin{aligned}
& \sum_{k, l=0,0}^{\infty, \infty}\left|a_{m_{0}, n_{0}, k, l}\right|>M-\frac{1}{4}, \quad \sum_{\substack{0<l<l_{0}, k_{0} \leq k \leq \infty}}\left|a_{m_{0}, n_{0}, k, l}\right| \leq \frac{1}{4}, \\
& \sum_{\substack{l_{0} \leq l \leq \infty, 0<k<k_{0}}}\left|a_{m_{0}, n_{0}, k, l}\right| \leq \frac{1}{4}, \quad \sum_{k, l=l_{0}, k_{0}}^{\infty, \infty}\left|a_{m_{0}, n_{0}, k, l}\right| \leq \frac{1}{4} .
\end{aligned}
$$

Let $\left[m_{p-1}\right],\left[n_{q-1}\right],\left[k_{p-1}\right]$, and $\left[l_{q-1}\right]$ be four chosen strictly increasing index sequences with $p, q=1 \cdots i-1, j-1$ with $k_{0}=l_{0}>0$. Using the RH-regularity conditions we now choose $m_{i}>m_{i-1}$ and $n_{j}>n_{j-1}$ such that

$$
\begin{gathered}
\sum_{\substack{0 \leq k \leq k_{i-1}, 0 \leq l \leq \infty}}\left|a_{m_{i}, n_{j}, k, l}\right|<\frac{1}{2^{i+j}}, \quad \sum_{\substack{0 \leq l \leq l_{j-1}, k_{i-1}<k \leq \infty}}\left|a_{m_{i}, n_{j}, k, l}\right|<\frac{1}{2^{i+j}}, \\
\sum_{k, l=0,0}^{\infty, \infty}\left|a_{m_{i}, n_{j}, k, l}\right|>M-\frac{1}{2^{i+j}} .
\end{gathered}
$$

In addition, we also choose $k_{i}>k_{i-1}$ and $l_{j}>l_{j-1}$ such that

$$
\sum_{\substack{k_{i-1}<k<k_{i}, l_{j} \leq l \leq \infty}}\left|a_{m_{i}, n_{j}, k, l}\right|<\frac{1}{2^{i+j}} \quad \text { and } \quad \sum_{\substack{l_{j-1}<l<\infty, k_{i} \leq k \leq \infty}}\left|a_{m_{i}, n_{j}, k, l}\right|<\frac{1}{2^{i+j}} .
$$

Let us define $[x]$ as follows:

$$
x_{k, l}:= \begin{cases}\frac{\bar{a}_{m_{i}, n_{j}, k, l}}{\left|a_{m_{i}, n_{j}, k, l}\right|}, & \text { if } k_{i-1}<k<k_{i}, l_{j-1}<l<l_{j}, \text { and } a_{m_{i}, n_{j}, k, l} \neq 0 ; \\ 0, & \text { otherwise . }\end{cases}
$$

Consider the following:

$$
\begin{aligned}
\left|y_{m_{i}, n_{j}}\right|= & \left|\sum_{k, l=0,0}^{\infty, \infty} a_{m_{i}, n_{j}, k, l} x_{k, l}\right| \geq-\sum_{\substack{0 \leq k \leq k_{i-1}, 0 \leq l \leq \infty}}\left|a_{m_{i}, n_{j}, k, l}\right| \\
& -\sum_{\substack{0 \leq l \leq l_{j-1}, k_{i-1}<k \leq \infty}}\left|a_{m_{i}, n_{j}, k, l}\right|-\sum_{\substack{k_{i-1}<k<k_{i}, l_{j} \leq l \leq \infty}}\left|a_{m_{i}, n_{j}, k, l}\right| \\
& -\sum_{\substack{l_{j-1}<l<\infty, k_{i} \leq k \leq \infty}}\left|a_{m_{i}, n_{j}, k, l}\right|+\sum_{\substack{l_{j-1}<l<l_{j}, k_{i-1}<k<k_{i}}} a_{m_{i}, n_{j}, k, l} \operatorname{sgn}\left(a_{m_{i}, n_{j}, k, l}\right)
\end{aligned}
$$




$$
\begin{aligned}
& \geq-\frac{1}{2^{i+j}}-\frac{1}{2^{i+j}}-\frac{1}{2^{i+j}}-\frac{1}{2^{i+j}}+M-5\left(\frac{1}{2^{i+j}}\right) \\
& =M-9 \frac{1}{2^{i+j}} .
\end{aligned}
$$

This implies that

$$
\mathrm{P}-\lim \sup [|y|] \geq M=M(\mathrm{P}-\lim \sup [|x|]) .
$$

Thus, if $A$ is real-valued then so is $[x]$ with $0<\limsup [x]<\infty$

$$
\mathrm{P}-\lim \sup [|y|]=M(\mathrm{P}-\lim \sup [|x|]) .
$$

THEOREM 3.2. If $A$ is a 4-dimensional matrix, then the following are equivalent

(1) For all real-valued double sequences $[x]$

$$
\mathrm{P}-\lim \sup [A x] \leq \mathrm{P}-\lim \sup [x]
$$

(2) A is an RH-regular summability matrix with

$$
\mathrm{P}-\lim _{m, n} \sum_{k, l=0,0}^{\infty, \infty}\left|a_{m, n, k, l}\right|=1 .
$$

Proof. To show that (1) implies (2), let $[x]$ be a bounded P-convergent double sequence, thus

$$
\mathrm{P}-\liminf [x]=\mathrm{P}-\lim \sup [x]=\mathrm{P}-\lim [x]
$$

and also

$$
\mathrm{P}-\limsup [A(-x)] \leq-(\mathrm{P}-\liminf [x]) .
$$

These imply that $\mathrm{P}-\liminf [x] \leq \mathrm{P}-\liminf [A x]$; thus

$$
\mathrm{P}-\liminf [x] \leq \mathrm{P}-\liminf [A x] \leq \mathrm{P}-\lim \sup [A x] \leq \mathrm{P}-\lim \sup [x] .
$$

Hence $[A x]$ is $\mathrm{P}$-convergent and $\mathrm{P}-\lim [A x]=\mathrm{P}-\lim [x]$. Therefore $A$ is an RH-regular summability matrix.

By Lemma 3.1 and its proof, there exists a bounded double sequence $[x]$ such that $\limsup [|x|]=1$ and P-limsup $[y]=A$, where $A$ is defined by $\left(\mathrm{RH}_{6}\right)$. This implies that

$$
1 \leq \mathrm{P}-\liminf _{m, n} \sum_{k, l=0,0}^{\infty, \infty}\left|a_{m, n, k, l}\right| \leq \mathrm{P}-\limsup _{m, n} \sum_{k, l,=0,0}^{\infty, \infty}\left|a_{m, n, k, l}\right| \leq 1,
$$

whence

$$
\mathrm{P}-\lim _{m, n} \sum_{k, l=0,0}^{\infty, \infty}\left|a_{m, n, k, l}\right|=1 .
$$

To prove that (2) implies (1) we show that if $[x]$ is a bounded P-convergent sequence and $A$ is an RH-regular matrix with

$$
\mathrm{P}-\lim _{m, n} \sum_{k, l=0,0}^{\infty, \infty}\left|a_{m, n, k, l}\right|=1,
$$


then

$$
\mathrm{P}-\lim \sup [A x] \leq \mathrm{P}-\lim \sup [x]
$$

For $p, q>1$ we obtain the following:

$$
\begin{aligned}
\left|\sum_{k, l=0,0}^{\infty, \infty} a_{m, n, k, l} x_{k, l}\right| & \\
= & \left|\sum_{k, l=0,0}^{\infty, \infty} \frac{\left|a_{m, n, k, l} x_{k, l}\right|-a_{m, n, k, l} x_{k, l}}{2}+\sum_{k, l=0,0}^{\infty, \infty} \frac{\left|a_{m, n, k, l} x_{k, l}\right|+a_{m, n, k, l} x_{k, l} \mid}{2}\right| \\
\leq & \sum_{k, l=0,0}^{\infty, \infty}\left|a_{m, n, k, l}\right|\left|x_{k, l}\right|+\sum_{k, l=0,0}^{\infty, \infty}\left(\left|a_{m, n, k, l}\right|-a_{m, n, k, l}\right)\left|x_{k, l}\right| \\
\leq & \|x\| \sum_{k, l=0,0}^{p, q}\left|a_{m, n, k, l}\right|+\|x\| \sum_{\substack{p<k<\infty, 0 \leq l \leq q}}\left|a_{m, n, k, l}\right| \\
& +\|x\| \sum_{\substack{0 \leq k<p, q<l<\infty}}\left|a_{m, n, k, l}\right|+\sup _{k, l>p, q}|x| \sum_{k, l>p, q}\left|a_{m, n, k, l}\right|+\|x\| \sum_{k, l=0,0}^{\infty, \infty}\left(\left|a_{m, n, k, l}\right|-a_{m, n, k, l}\right) .
\end{aligned}
$$

Using $\left(\mathrm{RH}_{1}\right)-\left(\mathrm{RH}_{4}\right)$ and

$$
\mathrm{P}-\lim _{m, n} \sum_{k, l=0,0}^{\infty, \infty}\left|a_{m, n, k, l}\right|=1,
$$

we take Pringsheim limits and get the desired result.

Acknowledgement. I am grateful to Professor J. A. Fridy for suggesting this problem and for many helpful discussions during this research. In addition I am grateful to the referee for his valuable comments.

\section{REFERENCES}

[1] R. P. Agnew, Abel transforms and partial sums of Tauberian series, Ann. of Math. (2) 50 (1949), 110-117. MR 10,291i. Zbl 032.15203.

[2] H. J. Hamilton, Transformations of multiple sequences, Duke Math. J. 2 (1936), 29-60. Zbl 013.30301.

[3] _ _ Change of dimension in sequence transformations, Duke Math. J. 4 (1938), 341342. Zbl 019.05901.

[4] _ A generalization of multiple sequence transformations, Duke Math. J. 4 (1938), 343-358. Zbl 019.05902.

[5]___ Preservation of partial limits in multiple sequence transformations, Duke Math. J. 5 (1939), 293-297. Zbl 021.22103.

[6] G. H. Hardy, Divergent Series, Oxford, at the Clarendon Press, 1949. MR 11,25a. Zbl 032.05801.

[7] K. Knopp, Zur Theorie der Limitierungsverfahren (Erste Mitteilung), Math. Z. 31 (1930), 115-127.

[8] I. J. Maddox, Some analogues of Knopp's core theorem, Internat. J. Math. Math. Sci. 2 (1979), 605-614. MR 81m:40012. Zbl 416.40004. 
[9] A. Pringsheim, Zur theorie der zweifach unendlichen Zahlenfolgen, Math. Ann. 53 (1900), 289-321.

[10] G. M. Robison, Divergent Double Sequences and Series, Trans. Amer. Math. Soc. 28 (1926), 50-73.

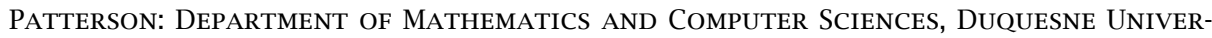
Sity, 440 College Hall, PitTsburgh, PA 15282, USA

E-mail address: pattersr@mathcs.duq.edu 


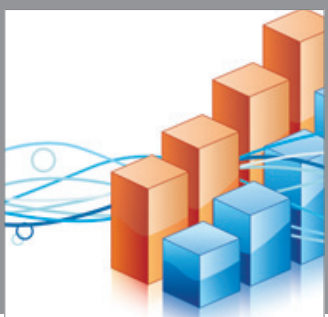

Advances in

Operations Research

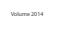

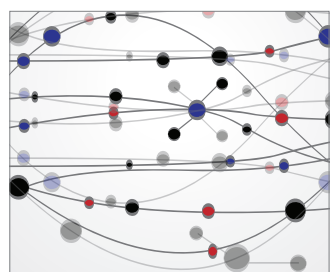

\section{The Scientific} World Journal
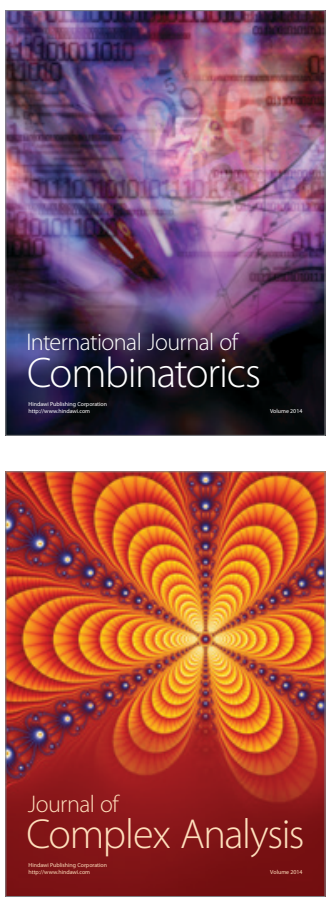

International Journal of

Mathematics and

Mathematical

Sciences
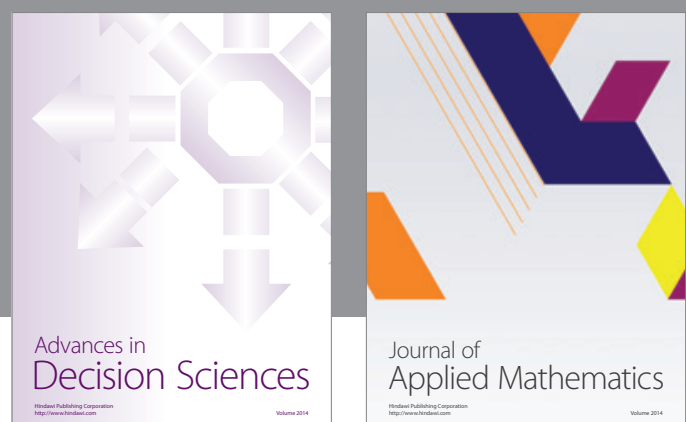

Journal of

Applied Mathematics
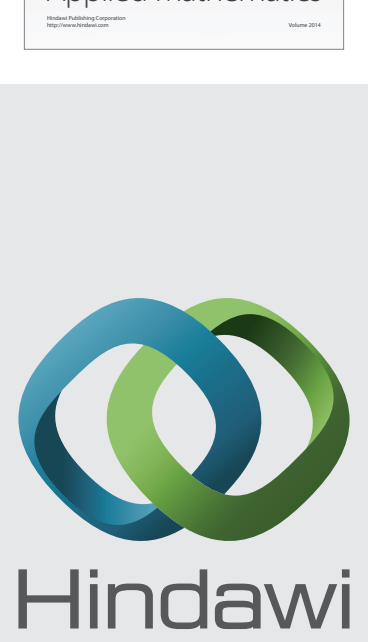

Submit your manuscripts at http://www.hindawi.com
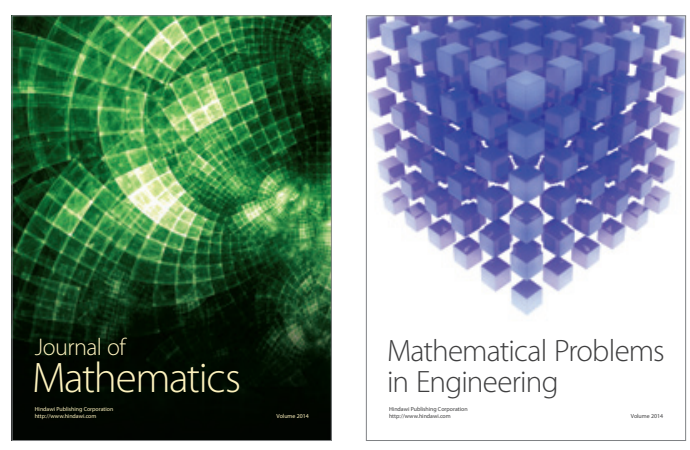

Mathematical Problems in Engineering
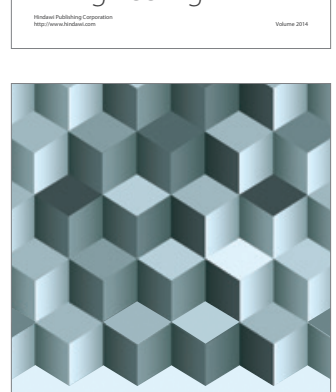

Journal of

Function Spaces
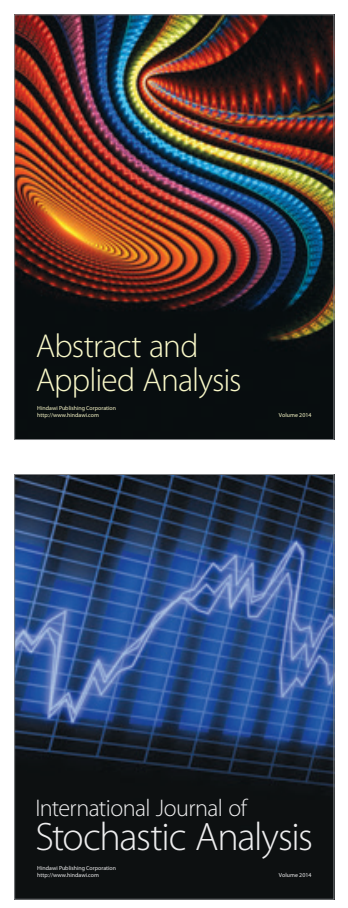

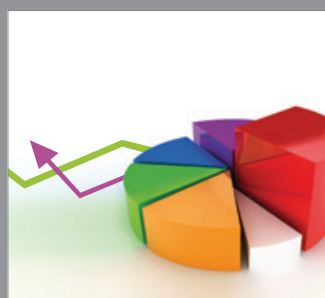

ournal of

Probability and Statistics

Promensencen
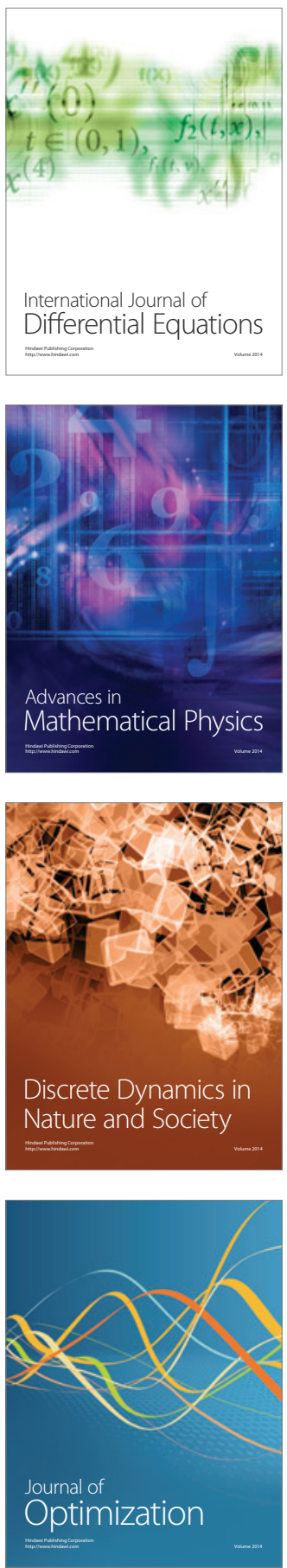